\title{
Classical and Recurrent Nova Models
}

\author{
Jordi José $^{1}$, Jordi Casanova ${ }^{1}$, Enrique García-Berro ${ }^{2}$, \\ Margarita Hernanz ${ }^{3}$, Steven N. Shore ${ }^{4}$, and Alan C. Calder ${ }^{5}$ \\ ${ }^{1}$ Dept. Física i Enginyeria Nuclear, Univ. Politècnica de Catalunya, E-08036 Barcelona \\ \& Institut d'Estudis Espacials de Catalunya, E-08034 Barcelona \\ email: jordi.jose@upc.edu \\ ${ }^{2}$ Dept. Física Aplicada, Univ. Politècnica de Catalunya, E-08860 Castelldefels (Barcelona) \\ \& Institut d'Estudis Espacials de Catalunya, E-08034 Barcelona \\ email: garcia@fa.upc.edu \\ ${ }^{3}$ Inst. Ciències de l'Espai (CSIC), Campus UAB, F. Ciències, E-08193 Bellaterra (Barcelona) \\ \& Institut d'Estudis Espacials de Catalunya, E-08034 Barcelona \\ email: hernanz@ieec.uab.es \\ ${ }^{4}$ Dipt. Fisica 'Enrico Fermi', Univ. Pisa \\ \& Ist. Nazionale di Fisica Nucleare, Sezione di Pisa, I-56127 Pisa \\ email: shore@df.unipi.it \\ ${ }^{5}$ Dept. Physics and Astronomy, Stony Brook Univ., Stony Brook, New York 11794-3800 \\ email: acalder@mail . astro.sunysb.edu
}

\begin{abstract}
Remarkable progress in the understanding of nova outbursts has been achieved through combined efforts in photometry, spectroscopy and numerical simulations. According to the thermonuclear runaway model, novae are powered by thermonuclear explosions in the hydrogen-rich envelopes transferred from a low-mass stellar companion onto a close white dwarf star. Extensive numerical simulations in 1-D have shown that the accreted envelopes attain peak temperatures ranging between $10^{8}$ and $4 \times 10^{8} \mathrm{~K}$, for about several hundred seconds, hence allowing extensive nuclear processing which eventually shows up in the form of nucleosynthetic fingerprints in the ejecta. Indeed, it has been claimed that novae can play a certain role in the enrichment of the interstellar medium through a number of intermediate-mass elements. This includes ${ }^{17} \mathrm{O},{ }^{15} \mathrm{~N}$, and ${ }^{13} \mathrm{C}$, systematically overproduced with respect to solar abundances, plus a lower contribution in a number of other species $(A<40)$, such as ${ }^{7} \mathrm{Li},{ }^{19} \mathrm{~F}$, or ${ }^{26} \mathrm{Al}$. At the turn of the XXI Century, classical novae have entered the era of multidimensional models, which provide a new insight into the physical mechanisms that drive mixing at the core-envelope interface.

In this review, we will present hydrodynamic models of classical novae, from the onset of accretion up to the explosion and ejection stages, both for classical and recurrent novae, with special emphasis on their gross observational properties and their associated nucleosynthesis. The impact of nuclear uncertainties on the final yields will be discussed. Recent results from 2-D models of mixing during classical nova outbursts will also be presented.
\end{abstract}

Keywords. accretion, accretion disks - convection - hydrodynamics — instabilities - nuclear reactions, nucleosynthesis, abundances — stars: novae, cataclysmic variables, white dwarfs.

\section{Introduction}

The Universe has evolved from a chemically poor stage (with only hydrogen, helium and traces of lithium) to an era dominated by the presence of a rich variety of chemical species, with nearly a hundred different stable nuclei. Stars, and in particular the suite of different nuclear processes operating at their interiors (and/or surfaces), have played a key role in the genesis of these new species, building blocks that have progressively clustered to form complex structures, including planets or even life forms. Stellar explosions, such 
as supernovae (both thermonuclear and core-collapse) or classical novae, have played a key role in this regard.

When the astronomical distance scale was not yet firmly established, both novae and supernovae were thought to be associated with the same explosive phenomenon, generically coined as "nova" (or "stella nova"). New light was shed by G.W. Ritchey, H.D. Curtis, H. Shapley, and others, who reported results from serendipitous discoveries of novae in the so-called spiral "nebulae" (which were actually galaxies) early in the XX Century. These scattered and heroic efforts were soon followed by systematic nova searches. Shapley and Curtis were among the first to question the real distances to spiral nebulae, presenting interesting arguments during their 1920 debate. A major step forward was achieved with nova $S$ Andromedae (S And), discovered and analyzed by the German astronomer Ernst Hartwig in 1885, as well as by the new distance scale of Hubble that placed Andromeda outside the Milky Way. This pushed nova $S$ Andromedae far away, at an incredible distance, and in turn with a huge intrinsic luminosity. Actually, Lundmark and Curtis, in the early 1920s, were the first to talk about "giant novae": It seems certain [...] than the dispersion of novae in spirals, and probably also in our galaxy may reach at least ten absolute magnitudes, as is evidenced by a comparison of $S$ And with the faint novae found recently in this spiral. A division into two magnitude classes is not impossible. Soon, it was clear that two different classes of stellae novae existed, the most luminous ones corresponding to what today we call supernovae.

Both novae and supernovae are characterized by a remarkable energy output, with peak luminosities reaching $\sim 10^{5}$ and $\sim 10^{10} \mathrm{~L}_{\odot}$, respectively. A basic difference between these explosive phenomena is the amount of mass ejected (i.e., the whole star, $\sim 1.4 \mathrm{M}_{\odot}$, in a thermonuclear supernova versus $10^{-4}-10^{-5} \mathrm{M}_{\odot}$ for a nova) as well as the mean ejection velocity $\left(>10^{4} \mathrm{~km} \mathrm{~s}^{-1}\right.$ in a supernova, and several $10^{3} \mathrm{~km} \mathrm{~s}^{-1}$ in a classical nova). This suggests that supernovae (rather than novae) are major players for the Galactic abundances. Nevertheless, the interest in novae extends much beyond their possible contribution to the Galactic chemical pattern: whereas the explosion that drives a supernova propagates supersonically (detonation), the ignition regime characteristic of a nova explosion is subsonic (deflagration). This makes the physics of classical novae complex and numerically challenging. Indeed, hydrodynamic effects (vorticity, turbulence, instabilities, etc.) combine with a suite of different nuclear processes in an envelope dominated by convective energy transport, operating at similar timescales.

\section{A Historical Side Note}

The understanding of the physics that lies behind a nova outburst has motivated large controversies and vivid discussions. One of the earliest suggestions for the possible origin of the 'stellae novae' appears in Newton's Principia Mathematica (book 3, prop. 42): So fixed stars, that have been gradually wasted by the light and vapors emitted from them for a long time, may be recruited by comets that fall upon them; and from this fresh supply of new fuel those old stars, acquiring new splendor, may pass for new stars. Indeed, the concept of revitalization of old stars (i.e., white dwarfs) by fresh supply of new fuel $\dagger$ (although not by comets!) is at the base of the widely accepted thermonuclear runaway model for the nova explosion.

There has been a long way from the early, naked-eye observations of novae carried out by ancient astronomers to our current understanding of the phenomenon, which relies on

$\dagger$ The idea of accretion emerged in Astrophysics around the XIX Century. See Clerke (1902). 
thermonuclear stellar explosions. Indeed, this has benefited from countless efforts, and particularly, from a number of observational breakthroughs:

- The application of spectroscopy to the study of nova explosions, pioneered by Huggins \& Miller (1866) on occasion of nova T CrB 1866.

- The discovery of neon in the spectra of GK Per (Sidgreaves 1901a,b) $\dagger$, pointing towards the existence of different nova classes (i.e., neon novae [with an underlying ONe white dwarf hosting the explosion] and non-neon novae [likely occurring on $\mathrm{CO}$ white dwarfs]).

- The identification of Doppler blueshifted lines in the ejecta (Campbell 1892, Clerke 1902).

- The early suggestion that some of the observed features in nova spectra could be due to ejection of a shell from a star (Pickering 1894).

- The link between the minimum in the DQ Her light curve and dust formation (Stratton \& Manning 1939).

- The discovery of the binary nature of DQ Her (Walker 1954), and the systematic studies of novae revealing that binarity is a common property of most cataclysmic variables (Kraft 1964; see also Sanford 1949 and Joy 1954).

A reasonable explanation of the physical nature of the burst, however, had to wait a few decades. Indeed, its thermonuclear origin was first theorized by Schatzmann (1949, 1951), although incorrectly attributed to nuclear fusion reactions involving ${ }^{3} \mathrm{He}$. The idea that CNO enhancement was critical for the explosion was proposed by Starrfield (1971a,b). Other notable contributions to the theoretical picture were made by Gurevitch \& Lebedinsky (1957) and Cameron (1959). Early attempts to mimic the explosion through the coupling of radiative transfer in an optically thick expanding shock with hydrodynamics can be found in Giannone \& Weigert (1967), Rose (1968) and Sparks (1969).

\section{Classical vs. Recurrent Novae}

Classical novae occur in short period $(1-12 \mathrm{hr})$, stellar binary systems, consisting of a compact white dwarf star (of, at most, $1.4 \mathrm{M}_{\odot}$ but planetary size) and a low-mass main sequence (i.e., a K-M dwarf) companion (although observations increasingly support the existence of more evolved secondaries, in some systems). Contrary to Type Ia supernovae, where the full white dwarf star gets disrupted by the strength of the explosion, classical nova outbursts are, roughly speaking, restricted to the outer, accreted envelopes. Hence, they are expected to recur since neither the star nor the binary system are disrupted by the event. Predicted recurrence times for nova outbursts are of the order of $10^{4}-10^{5} \mathrm{yr}$. (Notice, however, that in recurrent novae, likely powered by thermonuclear explosions on very massive white dwarfs, recurrence times range typically between $10-100 \mathrm{yr}$; it seems clear, though, that recurrence times must follow a nearly continuous sequence, ranging from the short values characteristic of [observed] recurrent novae to the long values predicted for classical novae.)

Novae have been observed in all wavelengths (but never detected so far in gammarays $\ddagger$, with an energy release that is only surpassed by supernova explosions and gammaray bursts. They constitute a very common phenomena (i.e., the second most frequent

$\dagger$ Sidgreaves only reported some unidentified spectral features, that were later understood to correspond to [Ne III] lines at 3869 and $3968 \AA$. See also Campbell (1895).

$\ddagger$ An unexpected emission at $>100 \mathrm{MeV}$ detected in the symbiotic binary V407 Cygni, attributed to shock acceleration in the ejected nova shell after interaction with the wind of the red giant, was reported by the Fermi-LAT Collaboration. See Abdo et al. (2010). 
type of stellar thermonuclear explosions in the Galaxy after Type I X-ray bursts); although just a few, 3 to 5 , are discovered every year (mainly by amateur astronomers), a much higher nova rate, around $30-40 \mathrm{yr}^{-1}$, has been predicted. The reason for the scarcity of detections in our Galaxy is extinction by interstellar dust.

About a dozen recurrent novae (by definition, a class composed of novae that have been seen in outburst more than once) have been identified so far. They are subdivided in two different categories: the very homogeneous class of long period binaries (like RS Oph), characterized by a red giant companion, and the heterogeneous class of short period binaries (which in turn can be spread in different subclasses defined by the recurrent novae U Sco, CI Aql and T Pyx. See Anupama 2002), formed by a white dwarf and a main sequence companion. Typical (observed) recurrence times range between 10 and $100 \mathrm{yr}$, which implies masses for the white dwarf hosting the explosion close to the Chandrasekhar limit, and also high mass-accretion rates around $10^{-7}-10^{-8} \mathrm{M}_{\odot} \mathrm{yr}^{-1}$. Hydrodynamic simulations of those systems suggest that not all the accreted material is ejected during the outburst, and hence the white dwarf star is expected to increase in mass. That makes recurrent novae possible candidate sources for type Ia supernovae.

\section{Nova Nucleosynthesis and Stellar Alchemy}

The early evolution of the thermonuclear runaway is dominated by the operation of both the proton-proton chains as well as the cold $\mathrm{CNO}$ cycle (i.e., ${ }^{12} \mathrm{C}(\mathrm{p}, \gamma){ }^{13} \mathrm{~N}\left(\beta^{+}\right){ }^{13} \mathrm{C}(\mathrm{p}$, $\left.\gamma)^{14} \mathrm{~N}\right)$. As the temperature increases, the characteristic timescale for proton captures onto ${ }^{13} \mathrm{~N}$ becomes shorter than its $\beta$-decay time, favoring a number of reactions of the hot CNO-cycle, such as ${ }^{13} \mathrm{~N}(\mathrm{p}, \gamma){ }^{14} \mathrm{O}$, together with ${ }^{14} \mathrm{~N}(\mathrm{p}, \gamma){ }^{15} \mathrm{O}$ and ${ }^{16} \mathrm{O}(\mathrm{p}, \gamma){ }^{17} \mathrm{~F}$. Since the envelope is degenerate, with a pressure depending only on the density rather than on temperature, the star cannot react with an expansion to the temperature increase (powered by the energy released by the suite of different nuclear processes). This paves the road for the explosive stage. Convection, that settles in the envelope when $\mathrm{T}$ exceeds $\sim 2 \times 10^{7} \mathrm{~K}$ ) plays a critical role in a nova explosion, carrying a substantial fraction of the short-lived, $\beta^{+}$-unstable nuclei ${ }^{14,15} \mathrm{O},{ }^{17} \mathrm{~F}\left({ }^{13} \mathrm{~N}\right)$, synthesized in the CNO-cycle, to the outer, cooler layers of the envelope. The energy released by these species during their decay powers the expansion and ejection stages of the outburst (Starrfield et al. 1972). In fact, the large amounts of ${ }^{14,15} \mathrm{O},{ }^{17} \mathrm{~F}$ and ${ }^{13} \mathrm{~N}$ synthesized during the outbursts translate into large amounts of their daughter nuclei ${ }^{15} \mathrm{~N},{ }^{17} \mathrm{O}$, and ${ }^{13} \mathrm{C}$ in the ejected shells (Figure 1).

Recurrent novae, in turn, are characterized by large hydrogen and helium abundances in the ejecta, with typical mass fractions around 0.6 and 0.4 , respectively (Hernanz \& José 2008). No large overproduction of metals with respect to solar values are found, in agreement with observations. This distinctive chemical pattern is interpreted as due to limited mixing episodes as well as to fast dynamic stages of the event. In particular, elements like Li, O or Ne are underproduced while S and Ar are slightly overproduced.

From the nuclear physics viewpoint, novae are unique stellar explosions: their nuclear activity, limited to about a hundred relevant species $(A<40)$ linked through a (few) hundred nuclear reactions, as well as the moderate temperatures achieved during the explosion $\left(10^{7}-4 \times 10^{8} \mathrm{~K}\right)$, allow us to rely primarily on experimental information (José, Hernanz \& Iliadis 2006). As shown in Figure 2, during classical nova outbursts the main nuclear path runs close to the valley of stability, and is driven by $(\mathrm{p}, \gamma),(\mathrm{p}, \alpha)$ and $\beta^{+}$ processes, with no significant contribution from any n- or $\alpha$-capture reaction.

The key role played by nuclear reactions has sparked a suite of different studies aimed at identifying the most critical reactions whose uncertainty has the largest impact on 

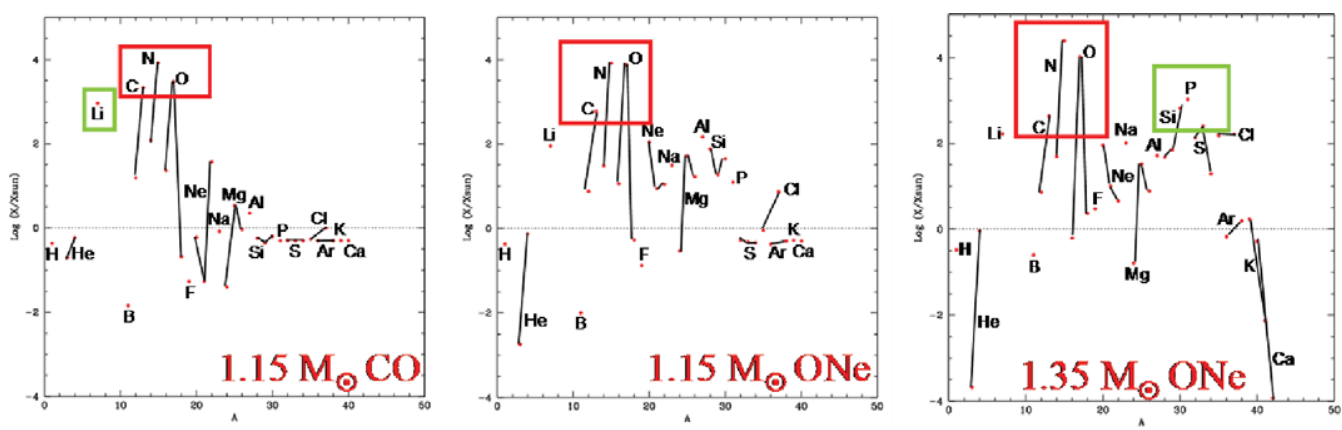

Figure 1. Main overproduction factors (mean abundances in the ejecta normalized over solar values) for models of nova outbursts on $1.15 \mathrm{M}_{\odot} \mathrm{CO}, 1.15 \mathrm{M}_{\odot} \mathrm{ONe}$, and $1.35 \mathrm{M}_{\odot} \mathrm{ONe}$ white dwarfs.

nova nucleosynthesis (see, for instance, Iliadis et al. 2002, for a sensitivity study based on 7350 network calculations). Many of the important reactions identified have been re-evaluated in recent years. Actually, the number of reactions whose uncertainty has still a strong impact on nova nucleosynthesis is small, being mainly dominated by the challenging reactions ${ }^{18} \mathrm{~F}(\mathrm{p}, \alpha){ }^{15} \mathrm{O},{ }^{25} \mathrm{Al}(\mathrm{p}, \gamma){ }^{26} \mathrm{Si}$, and ${ }^{30} \mathrm{P}(\mathrm{p}, \gamma){ }^{31} \mathrm{~S}$.

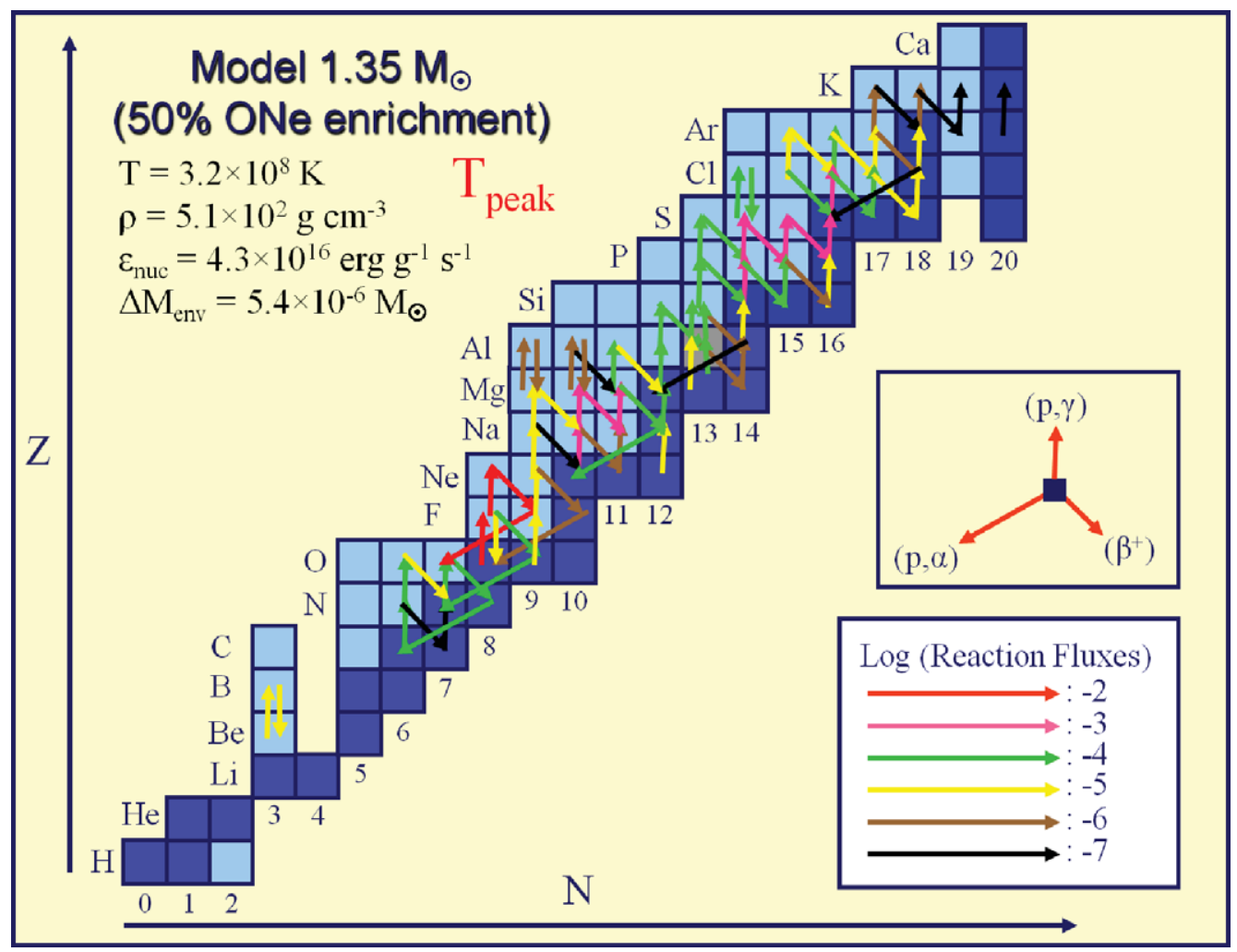

Figure 2. Main nuclear activity, shown in terms of reaction fluxes, at peak temperature, for a nova model involving a $1.35 \mathrm{M}_{\odot}$ white dwarf, with $50 \%$ ONe pre-enrichment. The dominant flow proceeds close to the valley of stability and is dominated by p-capture reactions and $\beta^{+}$-decays. Calculations have been performed with the 1-D, spherically symmetric hydrodynamic code SHIVA (see José \& Hernanz 1998). 
Current predictions, based on 1-D hydrodynamic models of nova outbursts, suggest that $\mathrm{Ca}$ is the likely nucleosynthetic endpoint, in agreement with observations of nova shells. There is, in general, good agreement between the abundance patterns inferred from observations and those derived from numerical hydro calculations. However, this statement requires a couple of remarks: first, the specific chemical abundance pattern spectroscopically inferred shows huge enhancements in metals, typically with mass fractions in the ejecta $\sim 0.5$ for neon novae and $\sim 0.25$ for non-neon novae. In order to match the observed abundances, 1-D models have (often) to artificially assume mixing between core material and the (solar-like) accreted envelope, since the temperatures achieved during the explosion do not allow significant CNO-breakout (see Sect. 5); and second, spectroscopic measurements of nova shells yield only atomic abundances, so comparison with theoretical predictions is rather limited in that respect.

Several species synthesized during classical nova outbursts provide potentially detectable $\gamma$-rays: this includes ${ }^{13} \mathrm{~N}$ and ${ }^{18} \mathrm{~F}$, that power a prompt $\gamma$-ray emission at and below $511 \mathrm{keV}$ through electron-positron annihilation, as well as the longer lived ${ }^{7} \mathrm{Be}$ and ${ }^{22} \mathrm{Na}$, that decay when the envelope is optically thin to $\gamma$-rays, powering line emission at 478 and $1275 \mathrm{keV}$, respectively. ${ }^{26} \mathrm{Al}$ is another important radioactive isotope synthesized during nova outbursts, although only its cumulative emission can be observed because of its slow decay. Indeed, the contribution of novae to the Galactic content of ${ }^{26} \mathrm{Al}$ is expected to be small ( $\sim 20 \%)$. We refer the reader to Hernanz $(2008)$ for a review of past and current theoretical predictions of the $\gamma$-ray output from classical novae, initiated with the seminal paper by Clayton \& Hoyle (1974).

Better perspectives to constrain theoretical nucleosynthesis results are offered by laboratory analyses of presolar meteoritic grains. Infrared and ultraviolet observations have revealed dust forming episodes in the shells ejected during classical nova outbursts (Gehrz et al., 1998). Since the pioneering studies of dust formation in novae by Clayton \& Hoyle (1976) (a concept already suggested by Cameron in 1973), all efforts devoted to the identification of potential nova grains relied mainly on the search for low ${ }^{20} \mathrm{Ne} /{ }^{22} \mathrm{Ne}$ ratios (since noble gases, such as $\mathrm{Ne}$, do not condense into grains, ${ }^{22} \mathrm{Ne}$ was attributed to in situ ${ }^{22} \mathrm{Na}$ decay, a clear imprint of a classical nova explosion). Indeed, Clayton and Hoyle pointed out several isotopic signatures (large overproduction of ${ }^{13,14} \mathrm{C},{ }^{18} \mathrm{O},{ }^{22} \mathrm{Na},{ }^{26} \mathrm{Al}$ or ${ }^{30} \mathrm{Si}$ ), that may help in the identification of such nova candidate grains. Twenty-five years later, most of these signatures still hold, in view of our current understanding of nova explosions, except ${ }^{14} \mathrm{C}$, bypassed by the main nuclear path in novae, and ${ }^{18} \mathrm{O}$, slightly overproduced by novae although grains nucleated in this environment are expected to be much more anomalous in ${ }^{17} \mathrm{O}$.

A major step forward in the discovery of presolar nova candidate grains was achieved by Amari et al. (2001) (see also Amari 2002), who reported on several SiC and graphite grains, isolated from the Murchison and Acfer 094 meteorites, with an abundance pattern qualitatively similar to nova model predictions: low ${ }^{12} \mathrm{C} /{ }^{13} \mathrm{C}$ and ${ }^{14} \mathrm{~N} /{ }^{15} \mathrm{~N}$ ratios, high ${ }^{30} \mathrm{Si} /{ }^{28} \mathrm{Si}$, and close-to-solar ${ }^{29} \mathrm{Si} /{ }^{28} \mathrm{Si}$; and high ${ }^{26} \mathrm{Al} /{ }^{27} \mathrm{Al}$ and ${ }^{22} \mathrm{Ne} /{ }^{20} \mathrm{Ne}$ ratios for some of the grains. But in order to quantitatively match the grain data, one had to assume a mixing process between material newly synthesized in the nova outburst and more than ten times as much unprocessed, isotopically close-to-solar, material before grain formation. One possible source of dilution might be mixing between the ejecta and the accretion disk, or even with the outer layers of the stellar companion. Concerns about the likely nova paternity of these grains have been raised (Nittler \& Hoppe 2005), after three additional micron-sized SiC grains were isolated from the Murchison meteorite with similar trends, but also with additional imprints (mainly non-solar Ti features), from which a supernova origin cannot be excluded. The presence of $\mathrm{Ti}$ in these grains, 

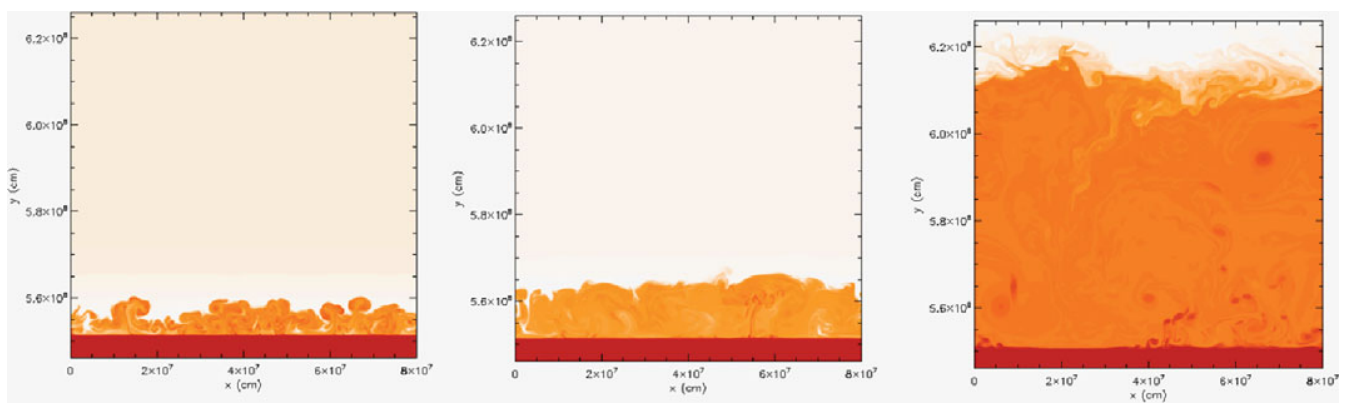

Figure 3. Two dimensional simulations of mixing at the core-envelope interface during a nova outburst in a CO white dwarf. Adapted from Casanova et al. (2010).

an element slightly above the canonical nucleosynthetic endpoint predicted for novae (Ca), has once more raised the issue of the possibility that other nuclear channels, like the CNO-breakout, may take place in nova outbursts under special circumstances. This, for instance, has been investigated in the context of slow white dwarf accretors in cataclysmic variables (Glasner \& Truran 2009), or in very-low metallicity systems (such as for primordial novae; see José et al. 2007).

\section{Multidimensional Nova Models: the Next Frontier}

Because of the moderate peak temperatures achieved during nova explosions, it is unlikely that the large metallicities inferred from the ejecta could result from thermonuclear processing of solar-like material. Instead, mixing at the core-envelope interface is a more likely explanation. Several mixing mechanisms have been suggested, including diffusion, shear or resonant gravity waves. The few multidimensional models of mixing at the coreenvelope interface available to date have proven particularly controversial. Indeed, two independent, two-dimensional studies (Glasner et al. 1997; Kercek et al. 1998), based upon the same 1-D initial model, reached totally different conclusions about the strength of the runaway and its capability to power a fast nova. The origin of such differences was carefully analyzed by Glasner et al. (2005), who concluded that the early stages of the explosion, prior to the onset of the TNR -when the evolution is almost quasi-staticare extremely sensitive to the outer boundary conditions. Recent efforts to disentangle this controversy have been performed with the hydrodynamic code FLASH. The results, reported in Casanova et al. (2010, 2011), show that the onset of Kelvin-Helmholtz instabilities in the plasma drives efficient mixing through the core-envelope interface. The mean metallicity achieved in the envelope, $\mathrm{Z} \sim 0.20-0.30$, is in agreement with the observed values, hence demonstrating the feasibility of this mechanism. These preliminary studies will be soon extended by means of $2-\mathrm{D}$ and $3-\mathrm{D}$ simulations of mixing for both classical and recurrent novae, currently underway.

This work has been partially supported by the Spanish grants AYA2010-15685 and AYA2008-04211-C02-01, by the E.U. FEDER funds, and by the ESF EUROCORES Program EuroGENESIS through the MICINN grant EUI2009-04167.

\section{References}

Abdo, A. A., et al. 2010, Science, 329, 817

Amari, S. 2002, New Astron. Rev., 46, 519 
Amari, S., Gao, X., Nittler, L., Zinner, E., José, J., Hernanz, M., \& Lewis, R. 2001, ApJ, 551, 1065

Anupama, G. C. 2002, in Classical Nova Explosions, ed. M. Hernanz \& J. José, New York, AIP, 32

Cameron, A. G. W. 1959, ApJ, 130, 916

Cameron, A. G. W. 1973, in Interstellar Dust and Related Topics, ed. J. M. Greenberg \& H. C. Van de Hulst, Dordrecht, Reidel, 545

Campbell, W. W., 1892, Astron. Nach., 131, 201

Campbell, W. W., 1895, ApJ, 1, 49

Casanova, J., José, J., Garcı a-Berro, E., Calder, A., \& Shore, S. N. 2010, A $\& A$, 513, L5

Casanova, J., José, J., Garcı a-Berro, E., Calder, A., \& Shore, S. N. 2011, A\&̛A, 527, A5

Clayton, D. D. \& Hoyle, F. 1974, ApJ, 187, L101

Clayton, D. D. \& Hoyle, F. 1976, ApJ, 203, 490

Clerke, A. M. 1902, Problems of Astrophysics, London, Nelson

Gehrz, R. D., Truran, J. W., Williams, R. E., \& Starrfield, S. M. 1998, PASP, 110, 3

Giannone, P. \& Weigert, A. 1967, Z. Astroph., 67, 41

Glasner, S. A., Livne, E., \& Truran, J. W. 1997, ApJ, 475, 754

Glasner, S. A., Livne, E., \& Truran, J. W. 2005, ApJ, 625, 347

Glasner, S. A. \& Truran, J. W. 2009, ApJ, 692, L58

Gurevitch, L. Z. \& Lebedinsky, A. I. 1957, in Non-stable stars, ed. G.H. Herbig, Cambridge Univ. Press: Cambridge, 77

Hernanz, M. 2008, in Classical Novae, M. Bode and A. Evans (eds.), Cambridge University Press, Cambridge, 252

Huggins, W. \& Miller, W. A. 1866, MNRAS, 26, 215

Iliadis, C., Champagne, A., José, J., Starrfield, S., \& Tupper, P. 2002, ApJS, 142, 105

José, J., Garcia-Berro, E., Hernanz, M., \& Gil-Pons, P. 2007, ApJ, 662, L103

José, J. \& Hernanz, M. 1998, ApJ, 494, 680

José, J., Hernanz, M., \& Iliadis, C. 2006, NPA, 777, 550

Joy, A. H. 1954, ApJ, 120, 377

Kercek, A., Hillebrandt, W., \& Truran, J. W. 1998, A\&A, 337, 379

Kraft, R. P. 1964, ApJ, 139, 457

Nittler, L. R. \& Hoppe, P. 2005, ApJ, 631, L89

Pickering, W. H. 1895, Observatory, 234, 436

Rose, W. K. 1968, ApJ, 152, 245

Sanford, R. F. 1949, ApJ, 109, 81

Schatzman, E. 1949, Ann. dAp., 12, 281

Schatzman, E. 1951, Ann. dAp., 14, 294

Sidgreaves, W. 1901a, MNRAS, 62, 137

Sidgreaves, W. 1901b, ApJ, 14, 366

Sparks, W. M. 1969, ApJ, 156, 569

Starrfield, S. 1971a, MNRAS, 152, 307

Starrfield, S. 1971b, MNRAS, 155, 129

Starrfield, S., Truran, J. W., Sparks, W. M., \& Kutter, G. S. 1972, ApJ, 176, 169

Stratton, F. J. M. \& Manning, W. H. 1939, Atlas of Spectra of Nova Hercules 1934, Cambridge, Solar Physics Observatory

Walker, M. F. 1954, PASP, 66, 230 